\title{
Effect of Cubic Liquid Crystalline Systems on Skin Localization of Oregonin and Hirsutanonol
}

\author{
Tae Jong Im, Myung Joo Kang, Dong Woo Seo and Jaehwi LeE* \\ Division of Pharmaceutical Sciences, College of Pharmacy, Chung-Ang University, Seoul 156-756, Korea
}

(Received July 22, 2008; Revised September 1, 2008; Accepted September 2, 2008)

\begin{abstract}
Monoolein-based cubic liquid crystalline systems were formulated for the local delivery of oregonin and hirsutanonol for the treatment of atopic dermatitis. The liquid crystalline phase and its nanodispersion containing drugs were prepared. The skin permeation and deposition properties of the drugs were examined in normal and delipidized rat skin. The proportion of oregonin (\%) deposited in normal skin after topical administration of the drugs in the form of aqueous solution, cubic phase or cubic nanodispersions were $1.53 \pm 0.46,3.62 \pm$ 0.17 and $5.13 \pm 0.73$, and those of hirsutanonol were $2.46 \pm 0.02,5.44 \pm 0.27$ and $17.28 \pm 2.19$, respectively. The greater lipophilicity and thus greater skin affinity of hirsutanonol than oregonin contributed the greater amount of skin deposition. The monoolein-based liquid crystalline phases significantly increased the amount of both drugs permeated and deposited. Approximately 3.2, 2.1 and 3.0 times greater amount of oregonin, and 3.4, 2.1 and 2.2 times greater amount of hirsutanonol were deposited in delipidized skin after administration of each drug in the form of aqueous solution, cubic phase and cubic nanodispersions system, respectively, because of lowered barrier function of the delipidized skin. In this study, the effects of drug property, vehicles type and skin condition on skin deposition and permeation properties of drug were examined and concluded that monoolein-based liquid crystalline systems would be a promising formulation for the local delivery of drugs.
\end{abstract}

Key words: Oregonin, Hirsutanonol, Cubic liquid crystalline phase, Cubic nanodispersion, Skin Localization

\section{INTRODUCTION}

Atopic dermatitis is a chronic inflammatory skin disease, occurring most commonly during early infancy and childhood, characterized by a chronically relapsing course. The skin of a patient with atopic dermatitis reacts abnormally and easily to irritants, food, and environmental allergens and becomes red, flaky and very itchy. Once that has been established, topical treatments focusing on reducing both the dryness and inflammation of the skin have primarily been used (Leung and Bieber, 2003).

Oregonin ((5S)-1,7-bis-(3,4-dihydroxyphenyl)-heptane3-one-5-O-beta-D-xylopyranoside, Fig. 1A) isolated from the bark of Alnus japonica is a diarylheptanoid derivative and hirsutanonol ((5S)-1,7-bis-(3,4-dihydroxyphenyl)-5hydroxyheptane-3-one, Fig. 1B) is an aglycone form of oregonin. Recently, it was reported that diarylheptanoids have a therapeutic effect on the dermal inflammation as

*Corresponding author

Tel: +82-2-820-5606, Fax: +82-2-816-7338

E-mail: jaehwi@cau.ac.kr selective 5-lipoxygenase inhibitor and thus are expected to be applicable for atopic dermatitis (Lee et al., 2003).

For the effective treatment of atopic dermatitis with topical administration of therapeutics, the penetration and<smiles>[M]OC(CCc1ccc(O)c(O)c1)CC(=O)CCc1ccc(O)c(O)c1</smiles>

(A)<smiles>O=C(CCc1ccc(O)c(O)c1)CC(O)CCc1ccc(O)c(O)c1</smiles>

(B)

Fig. 1. The chemical structures of oregonin (A) and hirsutanonol (B) 
accumulation of the agent into the relevant layers of the skin is required. To achieve this, monoolein-water systems have received considerable attention within the pharmaceutical field as it was demonstrated that monoolein itself is a penetration enhancer by promoting ceramide extraction and enhancement of lipid fluidity in the stratum corneum (Ogiso et al., 1995). The cubic crystalline phase has a highly viscous and ordered bicontinuous structure with curved lipid bilayers extending in three dimensions separated by water channels. The cubic crystalline phase is stable in excess water and can be fragmented into dispersions of cubic particles using adequate surfactants (Lindström et al., 1981). Aqueous dispersions of cubic nanoparticles are preferred as it is easier to administer than the very viscous cubic phase and smaller size of the vehicles shows the enhancement of penetration and deposition of the vehicles in the skin.

The aims of the present study were to formulate cubic crystalline phase or its nanoparticle system of oregonin and hirsutanonol and to investigate the localization factors for local skin delivery of oregonin and hirsutanonol. The effects of lipophilicity of drug, vehicle type and skin condition on skin permeation and deposition of the drugs were evaluated using the rat skin mounted in a Franz diffusion cells.

\section{METERIALS AND METHODS}

\section{Materials}

Oregonin and hirsutanonol were kindly gifted from Chung-Ang University Pharmacognosy Laboratory (Seoul, Korea). Phosphate buffered saline tablet was purchased from Sigma Chemical Company (St. Louis MO, USA) and glyceryl monooleate was purchased from Danisco A/S (RYLO ${ }^{\circledR}$ MG 19 PHARMA, Copenhagen, Denmark). Other chemicals and solvents were of analytical grade and used without further purification. Deionized water was prepared by the NANOpure Infinity ${ }^{\mathrm{TM}}$ from Barnstead International (USA).

\section{Preparation of Cubic liquid crystalline phase}

Pre-warmed aqueous solutions of oregonin or hirsutanonol were added to molten monoolein $\left(42^{\circ} \mathrm{C}\right)$ and thereafter, were allowed to stand in closed vials for 1 week at room temperature. The cubic phase system was composed of monoolein: drug: water at a ratio of 70:1:29 by weight (Lopes et al., 2006).

\section{Preparation of cubic nanodispersion}

Cubic nanodispersion was prepared by liquid precursor technique (Spicer et al., 2001). Oregonin or hirsutanonol in water were added to molten monoolein under vortexing. Then ethanol was added to the mixture and liquid precursor (water: ethanol: monoolein $=2: 3: 5, \mathrm{w} / \mathrm{w}$ ) was prepared. Then the liquid precursor and poloxamer 407 were mixed and were added to distilled water at room temperature. The resulting coarse dispersion was sonicated to produce the microdispersion and homogenized in a microfluidizer M110S (Microfluidics, USA) at 5000 psi for 30 cycles at $40^{\circ} \mathrm{C}$. Final formulation of cubic nanodispersion is composed of water: ethanol: monoolein: poloxamer 407 of 91:2:5:1, w/w. Poloxamer 407 was added as a stabilizer to avoid aggregation of the cubic nanodispersion (Spicer et al., 2001).

\section{Measurement of the size distribution and zeta potential of cubic nanodispersion}

Physical characteristics of the cubic nanodispersions (size and size distribution, polydispersity index, and zeta potential) were determined by dynamic light scattering (DLS) method with Zetasizer Nano-ZS (Malvern instrument, Worcestershire, UK). The measurements were repeated three times for each sample.

\section{Measurement of partition coefficient of drugs}

The partitioning properties of drugs into lipidic layers were evaluated by an octanol/water biphasic system. Distilled water $(30 \mathrm{ml})$ was added to $120 \mathrm{ml}$ of $\mathrm{n}$-octanol and $60 \mathrm{ml}$ of n-octanol was added to $300 \mathrm{ml}$ of water, and stirred at $200 \mathrm{rpm}$ for $24 \mathrm{~h}$ to saturate each solution. Oregonin or hirsutanonol was dissolved in saturated $\mathrm{n}$ octanol and vortexed with saturated water for $12 \mathrm{~h}$. Obtained samples were analyzed simultaneously using HPLC. Partition coefficients were obtained using the equation below.

$$
\log \mathrm{P}_{\text {oct } / \text { wat }}=\log \left(\frac{[\text { solute }]_{\text {octanol }}}{[\text { solute }]_{\text {water }}}\right)
$$

\section{Preparation of rat skin}

Sprague-Dawley male rats (6 to 8 weeks old) were obtained from Hanlim Experimental Animal Center (Hwasung, Korea). The animals were sacrificed by overdose inhalation of chloroform. Hair on the dorsal side of the animals was removed with shaving razor in the direction of tail to head without damaging the skin. The shaven part of skin was excised and the adhering fat and subcutaneous tissue was removed. The excised skin was washed with distilled water and subsequently used. Delipidized skin was obtained from the rat skin treated with 1 $\mathrm{ml}$ of chloroform: methanol $(2: 1 \mathrm{v} / \mathrm{v})$ for $60 \mathrm{~min}$ in order to 
extract the lipids from the skin.

\section{In vitro rat skin permeation study}

The freshly excised normal skins or delipidized skins were mounted between the donor and receptor compartments of Franz diffusion cells with the stratum corneum facing the donor compartment. Each Franz cell had a diffusional surface area of $1.76 \mathrm{~cm}^{2}$ and the volume of receptor compartments was $11.0 \mathrm{ml}$. The temperature of the receptor compartment was maintained at $36.5^{\circ} \mathrm{C}$ with an external, constant-temperature circulator water bath. The receptor compartments was filled with phosphate buffer solution ( $\mathrm{pH} 7.4)$ : ethanol $=70: 30(\mathrm{v} / \mathrm{v})$ and stirred at constant speed to mix the contents homogeneously. Aliquots $(500 \mu \mathrm{l})$ were withdrawn from the receptor compartment. Obtained samples were analyzed by HPLC.

\section{In vitro skin deposition study}

At the end of the permeation experiments $(12 \mathrm{~h})$, the skin surface was washed five times with buffer solution $(\mathrm{pH} 7.4)$ to remove excess drug from the surface. The skin was then cut into small pieces. The tissue was further homogenized with methanol solution for $5 \mathrm{~min}$. After shaking for $24 \mathrm{~h}$ and centrifuging for $10 \mathrm{~min}$ at $3000 \mathrm{rpm}$, the oregonin or hirsutanonol content was determined by HPLC.

\section{Assay and HPLC conditions}

The cumulative amount of drug permeated from excised rat skin into the receptor medium and deposited in excised rat skin was determined by HPLC. The HPLC system consisted of a quaternary pump (Hitachi, L-2130), an autosampler (Hitachi, L-2200), a column oven (Hitachi, L-2300), and a UV/VIS detector (Hitachi, L-2400, Japan) set to $280 \mathrm{~nm}$. The $\mathrm{C}_{18}$ column (150 mm $4.6 \mathrm{~mm}$ Capcell Pak, Shiseido, Tokyo, Japan) was used at $30^{\circ} \mathrm{C}$ and the mobile phase used was a mixtures of acetonitrile : water $(3: 7 \mathrm{v} / \mathrm{v})$ at a flow rate of $1 \mathrm{ml} / \mathrm{min}$. Sample of $20 \mu \mathrm{l}$ was injected onto the HPLC. Retention times of oregonin and hirsutanonol were $2.0 \mathrm{~min}$ and $3.6 \mathrm{~min}$, respectively.

\section{Statistical analysis}

All reported data are mean \pm SD. Statistical significance was checked by Student's $t$-test and considered to be granted at $P<0.05$, unless otherwise indicated.

\section{RESULTS AND DISCUSSION}

\section{Particle size and polydispersity of cubic nanodis- persion}

As shown in Table I, the average size of oregonin and
Table I. Particle size distribution and zeta potential of oregonin and hirsutanonol-loaded cubic nanodispersions (Mean $\pm S D$, $n=3)$.

\begin{tabular}{ccc}
\hline & Oregonin & Hirsutanonol \\
\hline Mean vesicular size $(\mathrm{nm})$ & $131.77 \pm 3.80$ & $94.20 \pm 1.67$ \\
Polydispersity index & $0.13 \pm 0.03$ & $0.12 \pm 0.02$ \\
Zeta potential $(\mathrm{mV})$ & $-4.81 \pm 0.30$ & $-3.33 \pm 0.34$ \\
\hline
\end{tabular}

hirsutanonol-loaded cubic nanodispersion were in the range of $131.77 \pm 3.80 \mathrm{~nm}$ (polydispersity index ; $0.13 \pm$ 0.03 ) and $94.20 \pm 1.67 \mathrm{~nm}$ (polydispersity index ; $0.12 \pm$ 0.02 ), respectively. The particle size distributions with a low polydispersity index of less than 0.3 generally indicate a narrow size distribution of the cubic nanodispersion and consequently a homogeneous dispersion.

\section{Partition coefficient of drug}

The experimental values of the logarithm of partition coefficient $(\log \mathrm{P})$ of oregonin and hirsutanonol were -0.02 \pm 0.01 and $0.99 \pm 0.01$, respectively. From this result, oregonin is regarded as more hydrophilic than hirsutanonol. The reason why hirsutanonol is less hydrophilic may be because hirutanonol is aglycone form. It is thus expected that hirsutanonol is more permeable via the skin.

\section{In vitro skin permeation and deposition study}

Drug deposition is important in drug therapies for atopic dermatitis. However, to be deposited to skin layers, the penetration of drug should be occurred. Thus, we primarily examined the drug deposition and discussed along with drug permeation. The results of in vitro permeation and deposition study of oregonin and hirsutanonol in normal skin and delipidized skin were shown in Fig. 2 and 3 , respectively. The proportions (\%) of oregonin deposited in normal skin at $12 \mathrm{~h}$ after topical administration of drug in the form of aqueous solution, cubic phase or cubic nanodispersions were $1.53 \pm 0.46,3.62 \pm 0.17$ and $5.13 \pm 0.73$, respectively and those of hirsutanonol from the aqueous solution, cubic phase or cubic nanodispersions were $2.46 \pm 0.02,5.44 \pm 0.27$ and $17.28 \pm 2.19$, respectively. The deposited amount of drug was greater when topically administered as aglycone form and incorporation of drug in monoolein-based nanodispersion significantly increased the amount of drug deposited.

And the proportions (\%) of oregonin deposited in delipidized skin from the aqueous solution, cubic phase or cubic nanodispersions were $4.88 \pm 1.01,7.51 \pm 2.05$ and $15.37 \pm 0.39$, respectively and those of hirsutanonol from aqueous solution, cubic phase or cubic nanodispersions 


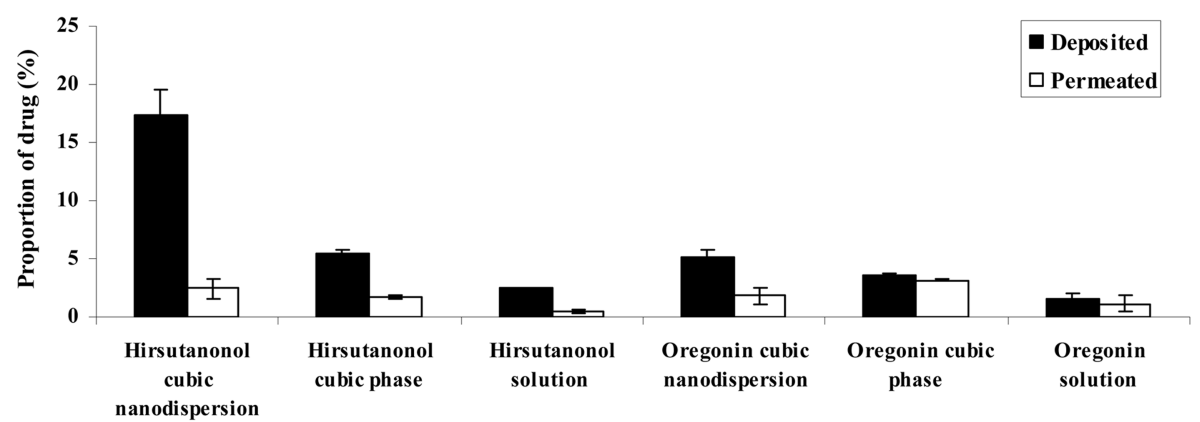

Fig. 2. The proportions (\%) of oregonin and hirstanonol permeated and deposited at $12 \mathrm{~h}$ after topical administration in normal rat skin (Mean $\pm S D, n=5)$.

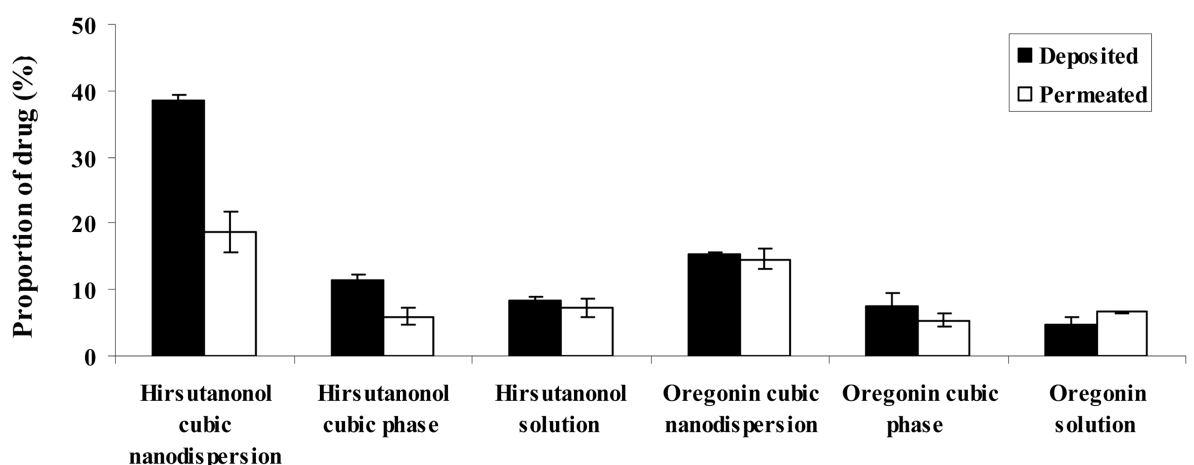

Fig. 3. The proportions (\%) of oregonin and hirsutanonol permeated and deposited at $12 \mathrm{~h}$ after topical administration in delipidized rat skin (Mean $\pm S D, n=5)$.

were $8.42 \pm 0.39,11.56 \pm 0.76$ and $38.62 \pm 0.88$, respectively. Approximately 3.2, 2.1 and 3.0 times greater amount of oregonin, and 3.4, 2.1 and 2.2 times greater amount of hirsutanonol were deposited in delipidized skin than normal skin after topical administration of each drug in the form of aqueous solution, cubic phase and cubic nanodispersions system, respectively.

\section{Effect of drug properties on drug localization in skin}

The deposited amount of drug was higher when topically administered as aglycone form hirsutanonol than administered as glycoside form oregonin in all transdermal vehicles. It is considered that the higher skin deposition of hirsutanonol was attributed to its greater lipophilicity than oregonin and, thus, leading to the higher affinity of hirsutanonol to the skin tissue. And the greater aqueous solubility of oregonin seemed to be attributed to hinder its deposition to the skin (Mitragotri, 2000).

\section{Effect of vehicle types on drug localization in skin}

Cubic nanodispersions and cubic bulk phase significantly $(p<0.05)$ increased the amounts of hirsutanonol and oregonin permeated and deposited for $12 \mathrm{~h}$ com- pared to the aqueous solution vehicle. The enhanced delivery of the drugs to the skin by monoolein-based systems might be attributed to the action of monoolein as penetration enhancer. Monoolein has demonstrated an increase in the delivery of various compounds to the skin and buccal tissues by increasing membrane fluidity or by extracting the lipid components (Ogiso et al., 1995; Carr et al., 1997; Lee and Kellaway, 2000). Particularly, the amount of hirsutanonol delivered to the skin layer (i.e., permeation and deposition) was significantly increased ( $p$ $<0.05$ ) at $12 \mathrm{~h}$ post-application when the nanodispersion was used as vehicles. The reason for this may be that the nanodispersion presents a larger surface area to interact with the skin and cubic nanodispersion itself can be acted as a penetrating enhancer owing to its minimal size.

The amount of oregonin and hirsutanonol in the receptor phase was quantified after $12 \mathrm{~h}$. The monoolein systems slightly increased the penetration of both oregonin and hirsutanonol compared to the aqueous solution vehicles, and thus relatively small fractions of the applied oregonin and hirsutanonol were delivered across the normal skin. Particularly, nanodispersion system of hirsutanonol 
showed the greatest skin deposition effects without significant increment of the amount permeated. As a result, it is speculated that the monoolein-based system, especially cubic nanodispersions, could improve the localization of oregonin and hirsutanonol within the skin.

\section{Effect of skin conditions on drug localization in skin}

It was reported that atopic dermatitis caused the loss of intercellular lipids such as cholesterol, ceramides and essential fatty acids - organized in multilayered lipid structures between the corneocytes in the intact barrier (Leung and Bieber, 2003). Therefore, the delipidized skin was used to mimic the skin conditions of atopic dermatitis. The same experiments were carried out using the topical formulations used in the normal skin. Approximately 2-3 times greater amount of orgonin and hirsutanonol were deposited in the skin when administered to delipidized skins. Increment in the amount of permeated across the skin and deposited to the skin might be attributed to the lowered barrier property of the delipidized skin. Monoolein systems, particularly nanodispersion system considerably increased the skin deposition and permeation of both oregonin and hirsutanonol in delipidized skin.

\section{CONCLUSIONS}

Monoolein-based liquid crystalline systems were formulated for the effective skin delivery of oregonin and hirsutanonol. And the effects of lipophilicity of drugs, vehicles type and skin conditions on the localization of drug were evaluated in normal and delipidized rat skin using Franz diffusion model. The greater lipophilicity and thus higher skin affinity of hirsutanonol than oregonin attributed the greater amount of skin. And monoolein systems, particularly nanodispersion system considerably increased the skin deposition and permeation of both oregonin and hirsutanonol in normal and delipidized skin. This study shows that topical delivery of oregonin and hirsutanonol could be significantly enhanced via monoolein-based systems.

\section{ACKNOWLEDGEMENTS}

This Research was supported by the Chung-Ang University Research Grants in 2007.

\section{REFERENCES}

Bender, J., Ericson, M. B., Merclin, N., Iani, V., Rosen, A., Engstrom, S. And Moan, J. (2005). Lipid cubic phases for improved topical drug delivery in photodynamic therapy. J. Control. Release 106, 350-360.

Carr, M.G., Corish, J. and Corrigan, O.I. (1997). Drug delivery from a liquid crystalline base across Visking and human stratum corneum. Int. J. Pharm. 157, 35-42.

Larsson, K. (2000). Aqueous dispersions of cubic lipid-water phases. Curr. Opin. Colloid Interface Sci. 5, 64-69.

Lee, J. and Kellaway, I. W. (2000). Buccal permeation of [DAla2, D-Leu5] enkephalin from liquid crystalline phases of glyceryl monooleate. Int. J. Pharm. 195, 35-38.

Lee, Y. A., Jeong, D. W., Kim, K. H., Kim, J. S., Kim, S. W. and Lee, M. W. (2000). Antioxidant activity of diarylheptanoids from the leaves of Alnus hirsute. Yakhak Hoeji 47, 193-196.

Leung, D. Y. and Bieber, T. (2003). Atopic dermatitis. Lancet 361(9352), 151-160.

Lindström, M., Ljusberg-Wahren, H., Larsson, K. and Borgström, B. (1981). Aqueous lipid phases of relevance to intestinal fat digestion and absorption. Lipids 16, 749-754.

Lopes, L. B., Lopes, J. L. C., Oliveira, D. C. R., Thomazini, J. A., Garcia, M. T. J., Fantini, M. C. A., Collett, J. H. and Bentlney, M. (2006). Liquid crystalline phases of monoolein and water for topical delivery of cyclosporin A: Characterization and study of in vitro and in vivo delivery. Eur. J. Pharm. Biopharm. 63, 146-155.

Mitragotri, S. (2000). Synergistic effect of enhancers for transdermal drug delivery. Synergistic effect of enhancers for transdermal drug delivery. Pharm. Res. 17, 1354-1359.

Ogiso, T., Masahiro, I. and Paku, T. (1995). Effect of various enhancers on transdermal penetration of indomethacin and urea and relationship between penetration parameters and enhancement factors. J. Pharm. Sci. 84, 482-488.

Spicer, P.T., Hayden, K. L., Lynch, M. L., Ofori-Boateng, A. and Burns, J. L. (2001). Novel process for producing cubic liquid crystalline nanoparticles (cubosomes). Langmuir. 17, 57485756. 